ACCEPTED MANUSCRIPT

\title{
Spectroscopy of buried states in black phosphorous with surface doping
}

To cite this article before publication: Zhesheng Chen et al 2020 2D Mater. in press https://doi.org/10.1088/2053-1583/ab8ec1

\section{Manuscript version: Accepted Manuscript}

Accepted Manuscript is "the version of the article accepted for publication including all changes made as a result of the peer review process, and which may also include the addition to the article by IOP Publishing of a header, an article ID, a cover sheet and/or an 'Accepted Manuscript' watermark, but excluding any other editing, typesetting or other changes made by IOP Publishing and/or its licensors"

This Accepted Manuscript is @ 2020 IOP Publishing Ltd.

During the embargo period (the 12 month period from the publication of the Version of Record of this article), the Accepted Manuscript is fully protected by copyright and cannot be reused or reposted elsewhere.

As the Version of Record of this article is going to be / has been published on a subscription basis, this Accepted Manuscript is available for reuse under a CC BY-NC-ND 3.0 licence after the 12 month embargo period.

After the embargo period, everyone is permitted to use copy and redistribute this article for non-commercial purposes only, provided that they adhere to all the terms of the licence https://creativecommons.org/licences/by-nc-nd/3.0

Although reasonable endeavours have been taken to obtain all necessary permissions from third parties to include their copyrighted content within this article, their full citation and copyright line may not be present in this Accepted Manuscript version. Before using any content from this article, please refer to the Version of Record on IOPscience once published for full citation and copyright details, as permissions will likely be required. All third party content is fully copyright protected, unless specifically stated otherwise in the figure caption in the Version of Record.

View the article online for updates and enhancements. 


\title{
Spectroscopy of buried states in black phosphorous with surface doping
}

Zhesheng Chen ${ }^{1}$, Jingwei Dong ${ }^{1}$, Christine Giorgetti ${ }^{1}$, Evangelos Papalazarou ${ }^{2}$, Marino Marsi'2, Zailan Zhang3,5, Bingbing Tian³, Qingwei Ma4, Yingchun Cheng ${ }^{4}$, JeanPascal Rueff ${ }^{5}$, Amina Taleb-Ibrahimi ${ }^{5}$, and Luca Perfetti ${ }^{*}$

${ }^{1}$ Laboratoire des Solides irradiés, CEA/DRF/IRAMIS, Ecole Polytechnique, CNRS, Institut Polytechnique de Paris, F-91128 Palaiseau, France

${ }^{2}$ Laboratoire de Physique des Solides, CNRS, Université Paris Saclay, Orsay 91405, France

${ }^{3}$ SZU-NUS Collaborative Innovation Center for Optoelectronic Science and Technology, International Collaborative Laboratory of 2D Materials for Optoelectronics Science and Technology of Ministry of Education, Institute of Microscale Optoelectronics, Shenzhen University, Shenzhen 518060, China

${ }^{4}$ Key Laboratory of Flexible Electronics \& Institute of Advanced Materials, Jiangsu National Synergetic Innovation Center for Advanced Materials, Nanjing Tech University, 30 South Puzhu Road, Nanjing 211816, China

${ }^{5}$ Société Civile Synchrotron SOLEIL, L'Ormedes Merisiers, Saint-Aubin, BP48, Gifsur-Yvette91192, France

*E-mail: luca.perfetti@polytechnique.edu

\begin{abstract}
Electrostatic gating or alkali metal evaporation can be successfully employed to tune the interface of layered black phosphorus (BP) from the semiconductor to a 2D Dirac semimetal. Although Angle Resolved Photoelectron Spectroscopy (ARPES)
\end{abstract}


experiments have captured the collapse of the band gap in the inversion layer, the quantitative estimate of the band structure evolution has been hindered by the short escape depth and matrix elements of the probed photoelectrons. Here, we precisely monitor the evolution of electronic states by time-resolved ARPES at photon energy of $6.3 \mathrm{eV}$. The probing depth of laser based ARPES is long enough to observe the buried electronic states originating from the valence band maximum. Our data show that the band gap has the maximal value of $0.32 \mathrm{eV}$ in the pristine sample and it shrinks down monotonically by increasing the carrier concentration in the topmost layer. Most interestingly, the band velocity of valence band increases by a factor two along the armchair direction, overcoming the value reported in graphene on silicon carbide (SiC). This control of band structure via external gating is of interest for the design of optoelectronic devices.

Keywords: black phosphorus; time-resolved ARPES; electron doping; band gap engineering; electronic structure

\section{Introduction}

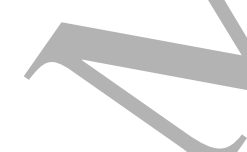

Van der Waals stacked black phosphorus (BP) has attracted interest in recent years because of the widely tunable band gap as function of thickness, ultrahigh carrier mobility in field effect transistors (FETs), and the possible emergence of topologically protected states ${ }^{1-5}$. It has been shown by high resolution angle resolved photoemission (ARPES) experiments that a large surface dipole induced via alkali atoms deposition can shift the relative position of the conduction and valence band above the inversion point of the electronic gap ${ }^{6-7}$. The crossover from insulator to Dirac semimetal ${ }^{8-9}$ leads to a pair of Weyl points with twofold degeneracy in the inversion layer. Recent reports suggest that such a transition can be reversibly controlled via gating techniques ${ }^{10}$. These findings highlight the potentials of BP as building block for functional devices based on exotics transport channels. Nonetheless, several controversial aspects on the band structure engineering ${ }^{6-7,11}$ via surface doping still need to be clarified. 
The easiest approach to observe the dispersion of electronic states in an accumulation layer is based on the combination of ARPES with in-situ evaporation of alkali atoms ${ }^{6-7,11-13}$. In the general case, a rigid band bending occurs at the very initial doping level whereas a band gap renormalization takes place upon strong electron doping. Due to the formation of the accumulation layer, the wave functions of conduction band and valence band spatially redistribute in the puckered layer structure. This process has extreme consequences when alkali atoms are deposited on the surface of black phosphorous. Despite the existing experiments, two limiting issues still challenge a quantitative analysis of the band gap collapse: 1) since BP is an intrinsic $p$-doped semiconductor ${ }^{14}$, conventional ARPES cannot be employed to extract the band gap until the chemical potential crosses the conduction band minimum. As a consequence, the doping dependent evolution of the band gap can be monitored only at high carrier concentration ${ }^{6-7}$. 2) As shown in Fig. 1, the surface dipole created by alkali metal evaporation pokes the wavefunction of the valence band towards the inner layers of $\mathrm{BP}^{15}$. It is therefore questionable whether a highly surface sensitive technique as ARPES can monitor the buried wavefunction of the valence band. As a matter of facts, previous ARPES experiments at 20-100 eV observed an anomalous band gap of $\sim 0.6 \mathrm{eV}$ at the initial stage of electronic doping ${ }^{6}$. This trend is not compatible with scanning tunneling spectroscopy measurements ${ }^{15}$ or ab-initio calculation of the band structure ${ }^{16}$, casting doubts on the electronic nature of electronic states that have been effectively probed.

In the present work, we overcome the aforementioned limits by performing timeresolved ARPES in pristine and in situ electron doped BP with probe photon energy centered at $6.3 \mathrm{eV}$. Time-resolved ARPES is a powerful technique that makes use of a pump-probe scheme to access the electronic states dispersion of the photoexcited surface 17-18. By theses means, we transiently occupy the conduction band of BP by an ultrafast pump pulse and measure the gap value with a subsequent probe pulse. Furthermore, the low photon energy of the probing beam results in an electron escape depth that is long enough to observe a reliable evolution of the buried 
valence band (see the calculated electron density in Fig. 1). Our data show that the bandgap monotonically shrinks from the pristine value of $0.32 \mathrm{eV}$ down to the inversion point. Most interesting, the Fermi velocity of the valence band displays a considerable enhancement. In the high doping regime, the holes moving along the highly dispersive direction acquire a band velocity larger than the one of graphene on silicon carbide (SiC). Such tunability of the electronic structure is expected also in the case of gated devices.

\section{Experimental details}

High-quality single crystals of BP (HQ Graphene) were cleaved and kept at the base pressure of $8 \times 10^{-11}$ mbar for all the duration of the experiment. The sample has been mounted on a cryogenic manipulator and oriented according to low energy electron diffraction patterns (LEED, see Supporting information). We deposited increasing doses of Cesium (Cs) atoms by a commercial getter source. The source has been carefully degassed and then approached to a distance of roughly $5 \mathrm{~cm}$ from the sample. The evaporation of the alkali metal is obtained by a resistive heating of the getter cartridge with the nominal current value. The deposition level leading to the inversion point corresponds to roughly 0.3 monolayers of absorbed Cs. Each step of alkali atoms deposition and Time-resolved ARPES measurement were performed while constantly keeping the sample at the temperature of $125 \mathrm{~K}$. No sample transfer or manipulation that could destabilize temperature or base pressure have been necessary between the subsequent deposition steps. All the experiments have been performed on the femto-ARPES setup, using a Ti: Sapphire laser system delivering $6 \mu \mathrm{J}$ Pulses with a $250 \mathrm{kHz}$ repetition rate ${ }^{19}$. Part of the laser beam ( $50 \mathrm{fs}$ pulse, $1.57 \mathrm{ev}$ ) is used to pump the sample while the rest is employed to generate the $6.3 \mathrm{eV}$ photons as probe pulse through cascade frequency mixing in $\mathrm{BaB}_{2} \mathrm{O}_{4}$ (BBO) crystals. The overall energy resolution of the experiment is $\cong 30 \mathrm{meV}$ and the cross correlation between pump and probe pulses has full width at half maximum (FWHM) of 150 fs. Time-resolved ARPES measurements have been performed with pump fluence of $230 \mu \mathrm{J} / \mathrm{cm}^{2}$ and pump probe delay time of 1 
picosecond. These parameters guaranty a transient occupation of the conduction band that is large enough in order to reliably extract the gap even in the pristine surface $^{17}$. Moreover, any photoinduced renormalization of band gap would be negligible at such moderate pump fluence ${ }^{17}$.

\section{Results and discussion}

The evaporation of alkali metals is a common approach to generate accumulation layers at the surface of semiconductors. Generally, the carrier concentration in the topmost layer is linearly dependent on the surface density of alkali atoms. Fig. 2 shows a series of photoelectron intensity maps of the photoexcited state as function of surface doping along the armchair and zigzag direction. We choose $p$-polarized photons for photoelectron intensity map along the armchair direction (panels a-h in Fig. 2). However, in the zigzag direction, the $p$-polarized probe couples strongly to the conduction band. As a consequence, we choose $p$-polarized photons when the occupation of the conduction band is weak (panels i-m in Fig. 2) while we polarize at $30^{\circ}$ with respect to $p$ orientation at higher filling level (panels n-p in Fig. 2). The wave vector asymmetry and drop of signal at the conduction band minimum are due to matrix elements of the photoemission process. The chemical potential $\mu_{\mathrm{e}}$ of BP in equilibrium conditions (pump off) is obtained by the reference spectrum of a metallic compound. Upon photoexcitation a steady state Surface PhotoVoltage (SPV) shifts the photoelectron intensity map with respect to the equilibrium case (see also the Supporting information). The internal reference $\mu$ (see white dashed line in the plots of Fig. 2 ) is given by $\mu_{\mathrm{e}}$ plus the SPV shift.

In agreement with previous finding, the alkali metal evaporation induces a surface dipole that dramatically restructures the electronic states in the accumulation layer. In Fig. 3, we plot the gap value $\Delta$ (energy of the conduction band minimum $E_{C B}$ minus energy valence band maximum $E_{V B}$, and also see the Supporting information for the positions of $E_{C B}$ and $E_{V B}$ ) as a function of the energy distance $\mu-E_{C B}$. Note that the band gap of BP keeps constant at $\sim 0.32 \mathrm{eV}$ as long as the chemical potential lies 
within the gap $\left(\mu-E_{C B}<0\right)$. In this regime the surface doping mainly compensates the acceptor states due to intrinsic vacancies. The electrons are transferred in the topmost layer of black phosphorus when $\mu-E_{C B}>0$ and the associated surface dipole induces a measureable shrinking of the bandgap for $\mu-E_{C B}>0.07 \mathrm{eV}$. Note that the initial $\Delta=0.32 \mathrm{eV}$ is in good agreement with scanning tunneling spectroscopy data acquired on a pristine surface ${ }^{15}$ and with infrared measurements of a bulk sample ${ }^{14}$. The band gap decreases linearly with $\mu-E_{C B}$ when the charge transfer keeps on filling the conduction band of the topmost layers. The inversion point $E_{C B}=E_{V B}$ takes place when $\mu-E_{C B}=0.3 \mathrm{eV}$, while $\Delta$ reaches the negative value of $-0.2 \mathrm{eV}$ at the largest doping level. We can estimate the electronic density in the accumulation layer from the area of the electronic pocket at a given filling of the conduction band (Luttinger theorem). The area of the ellipse $\pi k^{F} k^{F}{ }_{y}$ results in an electronic density of $4.5 \times 10^{13} 1 / \mathrm{cm}^{2}$ at the inversion point. This value is roughly a $50 \%$ lower than the critical surface density reported by ARPES experiments at higher photon energy7. In our opinion, the ARPES signal of Ref. 6,7 does not peak at the valence band maximum but is dominated by electronic states at higher binding energy. A manifold of such states exists because of the sizable dispersion of the valence band along the interlayer direction. These states would cross the conduction band at an electronic density higher than the real inversion point and may largely dominate the photoelectron intensity at $100 \mathrm{eV}$ if their electronic density extends nearer to the surface plane.

As shown by Fig. 3(a) the evaporation of potassium (K) instead of $\mathrm{Cs}$ atoms generate very similar results. It is interesting to compare in Fig. 3 the band gap evolution of our experiment with Scánning Tunneling Microscopy (STM) of a gated BP flake ${ }^{15}$. The agreement between the two different measurements is excellent if we assume that a stark field of $0.1 \mathrm{~V} / \mathrm{nm}$ displaces the chemical potential by $0.2 \mathrm{eV}$. Furthermore, our data are consistent with the monotonic decrease of gap value that has been reported by density-functional theory (DFT) calculations ${ }^{16}$. 
These favorable comparisons indicate that our experiment is indeed detecting the conduction band minimum and valence band maximum of the accumulation layer. The calculated local density of electronic states supports our claim. As shown in Fig. 1 , the square wavefunction of electronic states at the top of the $E_{V B}$ shifts by roughly $5 \mathrm{~nm}$ away from the surface upon the application of an external electric field of 0.1 $\mathrm{V} / \mathrm{nm}^{15}$. This distance is comparable to the escape depth of photoelectrons generated by $6.3 \mathrm{eV}$ photons whereas is 5 times longer than the photoelectron escape depth of a $\sim 100 \mathrm{eV}$ probing source. We have recently shown that topological states buried by absorbed molecules can be visible by laser based ARPES while they go undetected at higher photon energy ${ }^{20}$. By the same token, we think that the synchrotron based experiments ${ }^{6,7}$ could fail to observe the top of valence band of the accumulation layer.

In order to gain quantitative insights on the evolution of electronic states, we fit the dispersions along the armchair direction by the pseudorelativistic expression $\mathrm{E}=\mathrm{E}_{0} \pm\left(\delta+\sqrt{\mathrm{m}_{\mathrm{x}}{ }^{2} \mathrm{v}_{\mathrm{x}}{ }^{4}+\mathrm{v}_{\mathrm{x}}{ }^{2} \mathrm{p}^{2}}\right)$, where $\mathrm{m}_{\mathrm{x}}=\mathrm{m}_{\mathrm{x}}^{\mathrm{c}}\left(\mathrm{m}_{\mathrm{x}}=\mathrm{m}_{\mathrm{x}}^{\mathrm{v}}\right)$ is the effective mass for conduction band (valence band), $v_{x}=v_{x}^{c}\left(v_{x}=v_{x}^{v}\right)$ is the band velocity in the linear section of conduction band (valence band). $\mathrm{E}_{0}$ and $\delta$ are constants. Along the zigzag direction, the dispersion follows the parabolic form $E=E_{0} \pm\left(\Delta / 2+p^{2} / 2 m_{y}\right)$, where is the electronic gap $\Delta$, and $m_{y}=m_{y}^{c}\left(m_{y}=m_{y}^{v}\right)$ is the effective mass of the conduction band (valence band). As shown by the dotted lines in Fig. 2, the fitting curves reproduce with high accuracy the band dispersion along both crystallographic axes. The band gap turns out to be $2\left(\delta+\mathrm{m}_{\mathrm{x}} \mathrm{v}_{\mathrm{x}}{ }^{2}\right)$ in the armchair direction and $\Delta=2\left(\delta+\mathrm{m}_{\mathrm{x}} \mathrm{v}_{\mathrm{x}}{ }^{2}\right)$ is satisfied for each of the doping levels (with error bars of $\pm 0.03 \mathrm{eV}$ ). The/deformation of band dispersion upon electron doping is quantified by extracting fitting parameters for each band and high symmetry direction. Along zigzag direction, the fitting parameters $\mathrm{m}_{\mathrm{y}}$ does not depend on Cs deposition, neither for the conduction band $\left(\mathrm{m}_{\mathrm{y}}^{\mathrm{c}}=0.9 \pm 0.2 \mathrm{~m}_{\mathrm{e}}\right)$ nor for valence band $\left(\mathrm{m}_{\mathrm{y}}^{\mathrm{v}}=0.4 \pm 0.1 \mathrm{~m}_{\mathrm{e}}\right)$. Along armchair direction, the Cs evaporation does not modify the fitting in the conduction band parameter $\mathrm{m}_{\mathrm{x}}^{\mathrm{c}}=0.055 \pm 0.01 \mathrm{~m}_{\mathrm{e}}$ and 
$v_{x}^{c}=0.7 \pm 0.1 \times 10^{6} \mathrm{~m} / \mathrm{s}$. Nonetheless, Fig $2(\mathrm{a}-\mathrm{h})$ show a very interesting evolution of the valence band along the armchair direction. Figure 3(b-c) shows the values of $\mathrm{v}_{\mathrm{x}}^{\mathrm{v}}$ and $\mathrm{m}_{\mathrm{x}}^{\mathrm{v}}$ as a function of $\mu-E_{C B}$. Upon increasing doping level, the effective mass $\mathrm{m}_{\mathrm{x}}^{\mathrm{v}}$ shrinks below the detection limit while $\mathrm{v}_{\mathrm{x}}^{\mathrm{v}}$ increases by nearly two times (from $\mathrm{v}_{\mathrm{x}}^{\mathrm{v}}=0.7 \pm 0.1 \times 10^{6} \mathrm{~m} / \mathrm{s}$ to $\mathrm{v}_{\mathrm{x}}^{\mathrm{v}}=1.25 \pm 0.1 \times 10^{6} \mathrm{~m} / \mathrm{s}$ ). A similar tuning of / Fermi velocity has been previously achieved in graphene by employing substrates of different dielectric constant ${ }^{21}$. The maximal $v_{\mathrm{x}}^{\mathrm{v}}=1.25 \times 10^{6} \mathrm{~m} / \mathrm{s}$ value of this work is already higher than the Fermi velocity reported for graphene on SiC substrate and it is only a factor of two lower that the value reported in suspended graphene sheets ${ }^{22}$. The electron-electron interaction and the substrate polarizability explain the modified dispersion observed in graphene. Here instead, the formation of an accumulation layer shapes Fermi velocity and effective mass. Eventually, the anisotropic screening due to the self-organization of alkali metals should also be considered $^{23}$. The STM imaging of ref. 23 reveals that anisotropic charge screening from the surface layers induce the development of 1D potassium structures. Potassium atoms reorganize in short chains oriented along the armchair direction. Possibly, the potassium chains modify the electronic band dispersion in the accumulation layer, leading a larger Fermi velocity along the armchair direction. This hypothesis should be tested by ab-initio calculations of the electronic states in such specific configuration. Further theoretical and experimental investigations will be necessary in order to clarify this issue.

\section{Conclusions}

In conclusion, we performed Time resolved photoelectron spectroscopy of BP with surface doping. Awing for the larger escape depth of electrons emitted by a probe photon energy of $6.3 \mathrm{eV}$, we could observe the evolution of the buried valence band as a function of alkali metal deposition. Our data show that the band gap decreases monotonically from the bulk value of $0.32 \mathrm{eV}$ down to the inversion point. This finding is in excellent agreement with scanning tunneling spectroscopy measurements of gated BP and first principle calculations. A closer inspection 
reveals that the Fermi velocity of the electrons in the valence band increases by a factor of two upon doping the BP surface by Cs atoms. At the highest carrier concentration Fermi velocity of doped BP exceeds the value of the Dirac fermions in graphene on $\mathrm{SiC}$. This observation suggests that mobility of excited electrons in the accumulation layer of black phosphorous may considerably increase at large carrier density.

\section{Acknowledgement}

We thank DIM-Oximore and the Ecole polytechnique for funding under the project "ECO-GAN". We also acknowledge the financial support of the "ITEHR" contract (DGA, No. 201860 0074), of EU/FP7 under the contract Go Fast (No. 280555), of "Investissement d'Avenir" Labex PALM (ANR-10-LABX-0039-PALM), and of the China Scholarship Council (CSC, 201706170091).

\section{Supporting Information}

Low energy electron diffraction (LEED) on black phosphorus; The shift of conduction band and valence band as function of electron doping; Surface PhotoVoltage (SPV) and band bending as function of electron doping.

\section{References}

(1) Gusmão, R.; Sofer, Z.; Pumera, M. Black Phosphorus Rediscovered: From Bulk Material to Monolayers. Angew. Chem. 2017, 129, 8164-8185.

(2) Li, L.; Yu, Y.; Ye, G. J.; Ge, Q.; Ou, X.; Wu, H.; Feng, D.; Chen, X. H.; Zhang, Y. Black Phosphorus Field-Effect Transistors. Nat. Nanotech. 2014, 9, 372377.

Whitney, W. S.; Sherrott, M. C.; Jariwala, D.; Lin, W.-H.; Bechtel, H. A.; Rossman, G. R.; Atwater, H. A. Field Effect Optoelectronic Modulation of Quantum-Confined Carriers in Black Phosphorus. Nano Lett. 2016, 17, 78-84. 
(4) Doganov, R. A.; Farrell, E. C. T. O. R.; Koenig, S. P.; Yeo, Y.; Ziletti, A.; Carvalho, A.; Campbell, D. K.; Coker, D. F.; Watanabe, K.; Taniguchi, T.; et al. Transport Properties of Pristine Few-Layer Black Phosphorus by Van Der Waals Passivation in an Inert Atmosphere. Nat. Comm. 2015, 6, 1-7. Deng, B.; Tran, V.; Xie, Y.; Jiang, H.; Li, C.; Guo, Q.; Wang, X.; Tian, H.; Koester, S. J.; Wang, H.; Cha, J. J.; Xia, Q.; Yang Li; Black Phosphorus: a New Bandgap Tuning Knob. Nat. Comm. 2017, 8, 14474.

Kim, J. Observation of Tunable Band Gap and Anisotropic Dirac Semimetal State in Black Phosphorus. Science 2015, 349, 723.

Kim, J.; Huh, M.; Jung, S. W.; Ryu, S. H.; Sohn, Y.; Kim, K. S. Electronic Band Structure of Surface-Doped Black Phosphorus. J. Electron Spectrosc. 2017, 219, 86-91.

Baik, S. S.; Kim, K. S.; Yi, Y.; Choi, H. J. Emergence of Two-Dimensional Massless Dirac Fermions, Chiral Pseudospins, and Berry's Phase in Potassium Doped Few-Layer Black Phosphorus. Nano Lett. 2015, 15, 7788-7793.

Kim, J.; Baik, S. S.; Jung, S. W.; Sohn, Y.; Ryu, S. H.; Choi, H. J.; Yang, B.-J.; Kim, K. S. Two-Dimensional Dirac Fermions Protected by Space-Time Inversion Symmetry in Black Phosphorus. Phys. Rev. Lett. 2017, 119, 226801-226805.

Saito, Y.; Iwasa, Y. Ambipolar Insulator-to-Metal Transition in Black Phosphorus by Ionic-Liquid Gating. ACS Nano 2015, 9, 3192-3198.

Ehlen, N.; Sanna, A.; Senkovskiy, B. V.; Petaccia, L.; Fedorov, A. V.; Profeta, G; Grüneis, A. Direct Observation of a Surface Resonance State and Surface Band Inversion Control in Black Phosphorus. Phys. Rev. B 2018, $97,045143$.

Kang, M.; Kim, B.; Ryu, S. H.; Jung, S. W.; Kim, J.; Moreschini, L.; Jozwiak, C.; Rotenberg, E.; Bostwick, A.; Kim, K. S. Universal Mechanism of BandGap Engineering in Transition-Metal Dichalcogenides. Nano Lett. 2017, $17,1610-1615$.

(13) Zhang, Z.; Chen, Z.; Bouaziz, M.; Giorgetti, C.; Yi, H.; Avila, J.; Bingbing, T.; 
Shukla, A.; Perfetti, L.; Fan, D.; Li, Y.; Bendounan, A. Direct Observation of bang gap renormalization in layered Indium Selenide. ACS Nano 2019, 13 486-13491.

(20) Caputo, M.; Panighel, M.; Lisi, S.; Khalil, L.; Di Santo, G.; Papalazarou, E.; Hruban, A.; Konczykowski, M.; Krusin-Elbaum, L.; Aliev, Z. S.; Babanly, M. B.; Otrokov, M. M.; Politano, A., Chulkov, E. V.; Arnau, A.; Marinova, V.; Das, P. K.; Fujii, J.; Vobornik, I.; Perfetti, L.; Mugarza, A.; Goldoni, A.; Marsi M. Manipulating the Topological Interface by Molecular Adsorbates: Adsorption of Co-Phthalocyanine on $\mathrm{Bi}_{2} \mathrm{Se}_{3}$. Nano Lett. 2016 16, 34093414

(21) Hwang, C.; Siegel, D. A.; Mo, S.-K.; Regan, W. Ismach, A.; Zhang, Y.; Zettl, 
A.; Lanzara, A. Fermi velocity engineering in graphene by substrate modification. Scientific Reports 2012 2, 590. Elias, D. C. Gorbachev, R. V.; Mayorov, A. S.; Morozov, S. V., Zhukov, A. A.; Blake, P.; Ponomarenko, L. A.; Grigorieva, I. V.; Novoselov, K. S.; Guinea F.; Geim A. K. Dirac cones reshaped by interaction effects in suspended graphene. Nat. Phys. 2011 7, 701-704.

(23) Kiraly, B., Knol, E. J.; Volckaert, K.; Biswas, D.; Rudenko, A. N.; Prishchenko, Vladimir, D. A.; Mazurenko, G.; Katsnelson, M. I.; Hofmann, P.; Wegner, D.; and Khajetoorians, A. A. Anisotropic Two-Dimensional Screening at the Surface of Black Phosphorus. Phys. Rev. Lett. 2019, 123, 216403. 


\section{Figure 1}
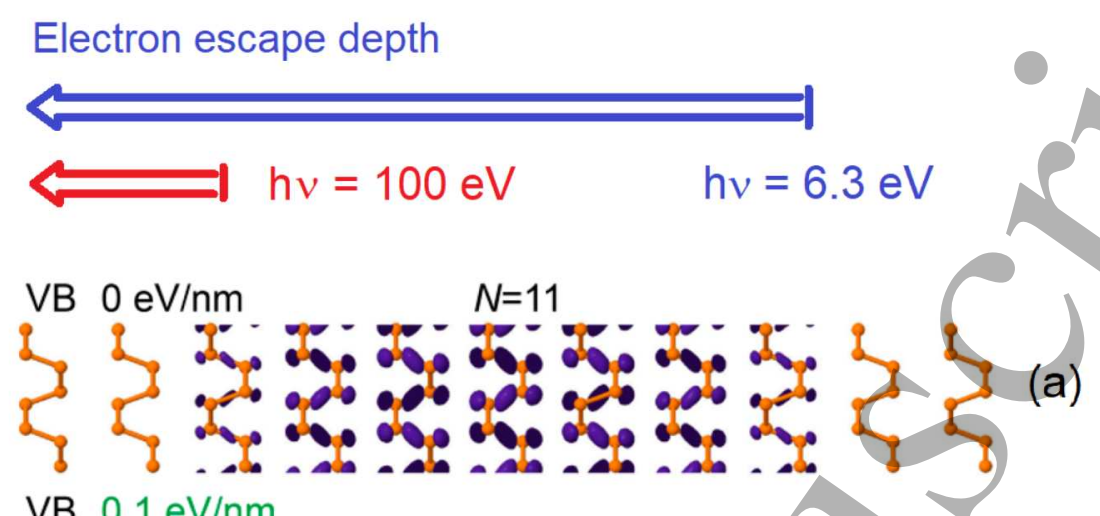

VB $0.1 \mathrm{eV} / \mathrm{nm}$

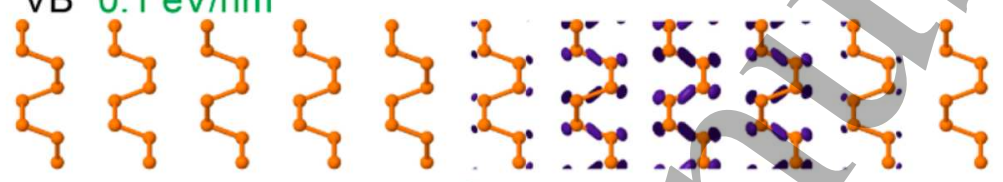

(b)

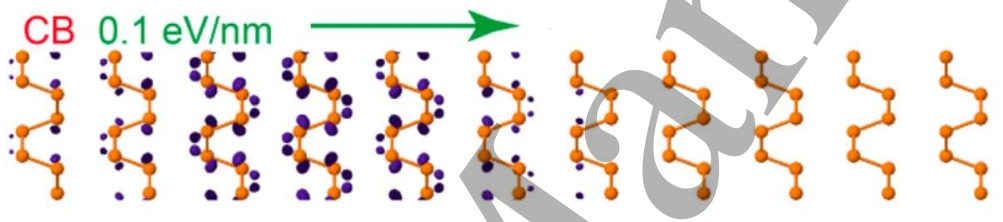

(c)

Figure 1. (a) Calculated spatial distribution of electronic density obtained by projecting the states at the top of the valence band on the atoms of a slab. (b) Puckering of valence band states after the application of $0.1 \mathrm{eV} / \mathrm{nm}$ field directed towards the right side of the slab. (c) Projected states of the conduction band minimum in the presence of the polarizing electric field. The blue and red arrows indicate the escape depth of photoelectrons probed by $6.3 \mathrm{eV}$ and $100 \mathrm{eV}$, respectively. This image has been readapted from the calculations and pictures of reference [15]. Copyright (2017) American Chemical Society. 


\section{Figure 2}
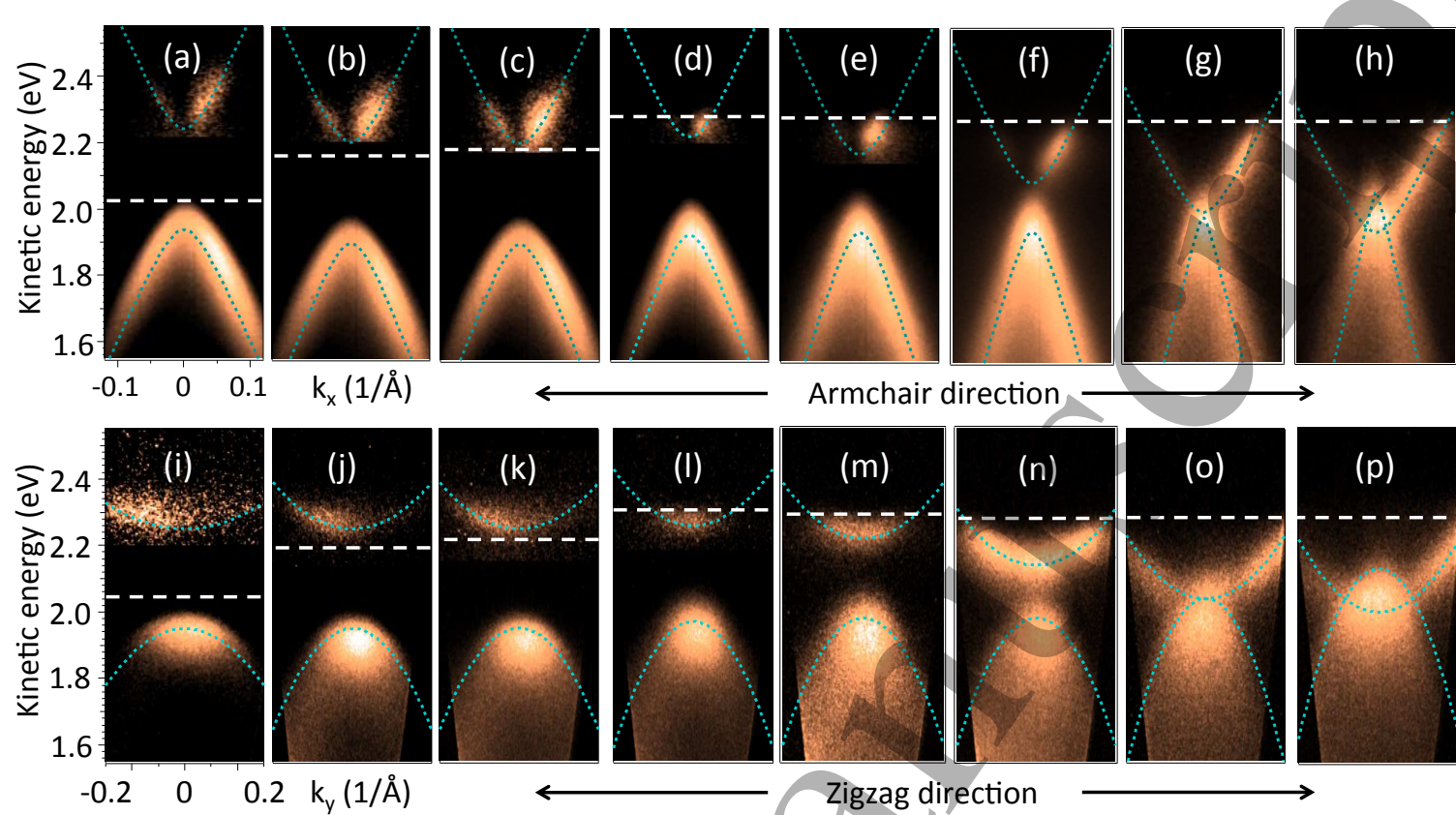

Figure 2: (a-h) Time-resolved ARPES data of BP along the armchair direction, as function of surface doping. (i-p) Time-resolved ARPES data of BP along the zigzag direction, as function of surface doping. The dashed lines indicate the position of chemical potential $\mu$. At low doping levels in (a-c) and (i-k), the gap has been visualized by measuring the photoelectron intensity maps at delay time of 1 ps after photoexcitation. At high doping levels in (d-h) and (l-p), the gap has been visualized by measuring the photoelectron intensity maps at negative delay. The conduction band signal of (a-e) and (i-l) has been multiplied by factors between 5 to 200, in order to equalize the intensity of the color scale. 


\section{Figure 3}
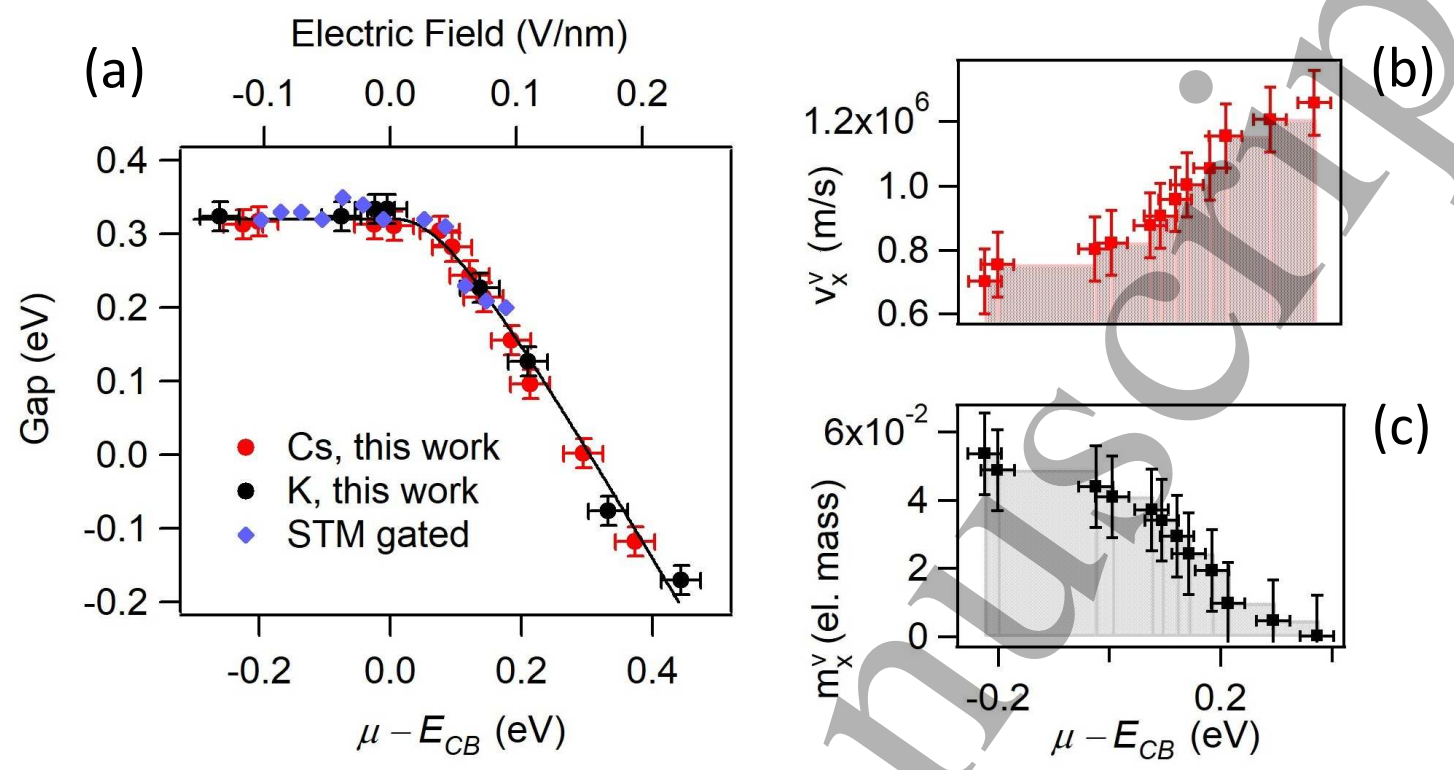

Figure 3: (a) Band gap at the surface of $\mathrm{BP}$ as function of surface doping by deposition of $\mathrm{Cs}$ atoms (red filled circles) and $\mathrm{K}$ atoms (black filled circles), respectively. The surface doping (bottom axis) is quantified by the difference between chemical potential $\mu$ and the minimum/of the conduction band energy $E_{C B}$. The solid line is a guide to the eye. As a term of comparison, the gap value (blue diamond) as function of polarizing field (top axis) is extracted from Ref. [15]. (b) The fermi velocity of the valence band along armchair direction and as function of doping level. (c) The effective mass of the valence band along armchair direction and as function of doping level. 